\title{
RF System for the SNS Accumulator Ring ${ }^{*}$
}

\author{
M. Blaskiewicz ${ }^{\dagger}$, \\ J.M. Brennan, J. Brodowski, J. Delong, M. Meth, K. Smith, A. Zaltsman \\ BNL, Upton NY 11973, USA
}

\section{Abstract}

During accumulation the RF beam current in the the Spallation Neutron Source ring rises from 0 to 50 Amperes. A clean, 250 nanosecond gap is needed for the extraction kicker risetime. Large momentum spread and small peak current are needed to prevent instabilities and stopband related losses. A robust RF system meeting these requirements has been designed.

\section{INTRODUCTION}

In broad terms, the Spallation Neutron Source consists of a linear accelerator, accumulator ring, and mercury target[1,2]. The linear accelerator produces a $1 \mathrm{GeV}, H^{-}$ beam which is charge exchange injected for $\approx 1000$ turns. After accumulation the beam is extracted using a fast kicker and sent to the mercury target. The purpose of the accumulator ring RF system is to maintain a gap for the rise time of the extraction kicker while maintaining low peak beam current and large momentum spread[3, 4, 5]. The latter considerations prevent space charge stopband related losses and coherent instabilities. Table 1 summarizes RF related machine parameters.

Table 1: Machine Parameters

\begin{tabular}{|c|c|}
\hline parameter & value \\
\hline circumference & $248 \mathrm{~m}$ \\
transition gamma & 5.25 \\
total h=1 voltage & $40 \mathrm{kV}$ \\
h=1 gap capacitance & $3 \mathrm{nF}$ \\
total h=2 voltage & $20 \mathrm{kV}$ \\
h=2 gap capacitance & $0.75 \mathrm{nF}$ \\
space charge $\mathrm{Z} / \mathrm{n}$ & $\mathrm{i} 200 \Omega$ \\
proton kinetic energy & $1 \mathrm{GeV}$ \\
injected bunch length & $610 \mathrm{~ns}$ \\
injected energy spread & $\pm 3.8 \mathrm{MeV}$, full \\
protons at extraction time & $2.08 \times 10^{14}$ \\
accumulation time & $1100 \mathrm{turns}$ \\
extraction gap & $250 \mathrm{~ns}$ \\
repetition rate & $60 \mathrm{~Hz}$ \\
\hline
\end{tabular}

\footnotetext{
* Work performed under the auspices of the United States Department of Energy

†blaskiewicz@bnl.gov
}

\section{CAVITY AND POWER AMPLIFIER}

Harmonic numbers of 1 and 2 imply RF frequencies of $f_{1}=1.05 \mathrm{MHz}$ and $f_{2}=2.11 \mathrm{MHz}$. Each cavity is composed of two RF gaps driven in parallel by the power amplifier. The $h=1$ cavities are designed for a gap voltage of $V_{g}=10 \mathrm{kV}$ and the $h=2$ cavities are identical but for less gap capacitance. Inductance is supplied by coaxial stacks of Phillips 4M2 ferrite. There are 21 rings per gap and each ring is $2.72 \mathrm{~cm}$ thick. The rings have inner and outer diameters of $25 \mathrm{~cm}$ and $50 \mathrm{~cm}$, respectively.

A large magnetic field in the ferrite cuts down on the total cavity length. The AGS Booster uses the same ferrite, the same 2 to 1 ratio of outer to inner radius, and a similar frequency range. Assuming the same RF magnetic field, an $h=1$ gap field of $13 \mathrm{kV}$ can be supported without Q losses or similar ferrite problems. Assuming the magnetic field varies as $1 / r$ within the ferrite, the magnetic field has a maximum RF amplitude of $B_{r f}=31 \mathrm{mT}$ for $V_{g}=10 \mathrm{kV}$. The cavity resonant frequency is tuned using bias current which flows in opposite directions through the two cells leading to negligible RF voltage across the bias supply. The baseline design calls for a constant resonant frequency during the cycle, but the possibility of dynamically tuning the cavity is a principle area of R\&D. The first article cavity has been built. Low power measurements of the resonant frequency and $R / Q$, with no bias, agreed with design values at the few percent level.

The design of the power amplifier is driven by beam loading requirements. During the millisecond of accumulation the average beam current rises from 0 to $34 \mathrm{~A}$. The maximum amplitude of the first harmonic component is $I_{1}=50 \mathrm{~A}$ and the second harmonic component is down by a factor of 7 . The philosophy here is to design the power amplifier for $h=1$ and use the same design for $h=2$. This reduces EDIA significantly and allows the $h=2$ system to to be easily modified to $h=1$ if desired. The base line design requires the power amplifier to fully compensate the beam current while providing the necessary quadrature component to drive the gap voltage. The cavity resonant frequency is fixed, and equal to the RF frequency. In some sense this may be pessimistic but the consequences of this assumption to the overall system cost are not great, whereas the benefits for system performance and reliability are very valuable.

A Thompson (TH558) tetrode drives two gaps in parallel while supplying the necessary anode current. With three cavities (6 accelerating gaps) at $h=1$ and $7 \mathrm{kV} /$ gap the anode dissipation reaches $500 \mathrm{~kW}$ at the end of accumula- 
tion. The time average dissipation is much smaller, about $50 \mathrm{~kW}$, but very high reliability is required and stressing the tube could lead to shorter life. The $h=2$ system has one cavity with two gaps and $10 \mathrm{kV} /$ gap. The $h=2$ beam loading is much smaller than for $h=1$.

Since the RF system is on for $\sim 2 \mathrm{~ms}$ every $16.7 \mathrm{~ms}$, the tube will be biased off for most of the time. The grid bias supply can switch between cutoff and quiessant states in $\approx 100 \mu \mathrm{s}$. An anode power supply consists of two, charging $14 \mathrm{kV}, 3 \mathrm{~A}$ supplies and capacitor bank. The total capacitance of the bank is $75 \mu \mathrm{F}$. For a $10 \mathrm{~A}$ draw starting at $10 \mathrm{kV}$, with a charging current of $6 \mathrm{~A}$ the anode voltage droops by $3 \%$ over $2 \mathrm{~ms}$.

Every effort is being made to reduce surprises during commissioning. Toward this end a full power beam-cavity simulator is planned. This device would use one power amplifier to drive the cavity, and another to simulate the beam. The beam circuit will be attached to the anode of the beam PA and encircle the ferrite stack of the cavity. The low level drive for the simulator will mimick both dipole and quadrupole beam oscillations. In this way we will test the tuning scheme, the high level RF system, and the low level RF system well before installation.

\section{VOLTAGE CONTROL AND BEAM STABILITY}

In analytic models of steady state beam loading $[6,7,8]$ the ratio of beam induced voltage to total gap voltage plays an important role. For SNS this beam loading parameter is $\sim 4$, so the stability if the RF system could be compromised. Unlike typical accelerators, the SNS cycle time is comparble to the synchrotron period, so the transient response of the beam-cavity interaction is very important. Additionally, the dual harmonic system leads to very nonlinear dynamics and analytic results are difficult to obtain. Therefore, simulations of the beam-cavity system have been a primary design tool.

A schematic of the beam, cavity and generator for a single gap cavity is shown in Figure 1. The electric field in the gap, $E_{g}$ is related to the gap voltage $V_{g}$ and the equivalent gap length $\ell_{g}$ via $E_{g} \ell_{g}=-V_{g}$. The beam current, $I_{b}$ is positive to the left so a positive value of $V_{g}$ accelerates the beam. Suppose the system is quiessant for $t<0$. The anode current $I_{A}$, gap voltage and beam current are related via

$$
\begin{aligned}
\frac{I_{A}(t)-I_{A}(0)}{n_{\text {gap }}}-I_{b}(t) & =\frac{V_{g}(t)}{R_{f}}+C_{g} \frac{d V_{g}(t)}{d t} \\
& +\frac{1}{L_{g}} \int_{0}^{t} V_{g}\left(t^{\prime}\right) d t^{\prime}
\end{aligned}
$$

where $C_{g}$ and $L_{g}$ are the equivalent capacitance and inductance of one gap, $R_{f}$ is the shunt impedance of the ferrite for one gap, and $n_{\text {gap }}=2$ is the number of gaps driven by one power amplifier. The anode voltage $V_{A}$, gap voltage, and anode supply voltage $B^{+}$are related via $V_{A}(t)+V_{g}(t)=B^{+}$. With only a few percent droop, the anode supply voltage was assumed constant. The final relation involves the anode current, grid drive voltage $V_{G}$ and anode voltage. For reasonable parameters the anode voltage depends only on the combination $V_{A}+\mu V_{G}$ where the amplification factor $\mu$ depends only on the screen voltage, which is held constant during the cycle. Figure 2 shows $I_{A}=G\left(V_{A}+\mu V_{G}\right)$ for the TH558 tetrode with a screen voltage of $2 \mathrm{kV}$.

The RF control loops directly affect the grid drive voltage so in equation (1) set

$$
I_{A}(t)=G\left(B^{+}-V_{g}(t)+\mu V_{G}(t)\right) .
$$

The grid drive voltage is taken to be

$$
V_{G}(t)=I_{G}(t) \sin \left(\omega_{r f} t\right)-Q_{G}(t) \cos \left(\omega_{r f} t\right)+\bar{V}_{G},
$$

where $\omega_{r f}$ is the ideal RF frequency, $\bar{V}_{G}$ is a DC offset and the in phase $I_{G}$ and quadrature $Q_{G}$ amplitudes vary slowly. The ideal RF frequency is set before injection begins and the phase is chosen so that extraction can be defined to better than one turn.

Since the beam current is not a pure sinusoid, the gap voltage is not a pure sinusoid. However, the in phase $I_{g}$ and quadrature $Q_{g}$ components of the gap voltage at $\omega_{r f}$ can be defined with respect to a given measurement procedure. The control system will be digital, so a natural unit of time is one turn. Define the raw in phase and quadrature components of the gap voltage on the $n$th turn to be

$$
\begin{aligned}
I_{g}^{0}(n) & =\frac{2}{T_{r f}} \int_{(n-1) T_{r f}}^{n T_{r f}} \sin \left(\omega_{r f} t\right) V_{g}(t) d t, \\
Q_{g}^{0}(n) & =-\frac{2}{T_{r f}} \int_{(n-1) T_{r f}}^{n T_{r f}} \cos \left(\omega_{r f} t\right) V_{g}(t) d t .
\end{aligned}
$$

In the actual system these might be generated by digital FIR filters but for any reasonable clock rate there will be little difference between the analog and digital quantities. The raw values are then subject to delay and smoothing to create the inputs to the control system. This is modeled as a lagged, auto-regressive filter,

$$
\begin{gathered}
I_{g}(n+1)=\delta I_{g}(n)+(1-\delta) I_{g}^{0}\left(n-n_{g}\right), \\
Q_{g}(n+1)=\delta Q_{g}(n)+(1-\delta) Q_{g}^{0}\left(n-n_{g}\right),
\end{gathered}
$$

where $\delta=\exp \left(-T_{r f} r_{g}\right), r_{g}$ is the accumulation rate, and $n_{g}$ is the signal delay in turns. The lower limit on the delay is set by the round trip time between the ring and the RF control room, $n_{g} T_{r f} \gtrsim 750 \mathrm{~ns}$.

In general the in phase and quadrature components of the gap voltage will not be equal to their target values $\hat{I}_{g}(n)$ and $\hat{Q}_{g}(n)$. The first level of RF control varies the grid drive amplitudes $I_{G}$ and $Q_{G}$ to approach the desired values of the gap voltage components $I_{g}$ and $Q_{g}$. To obtain 


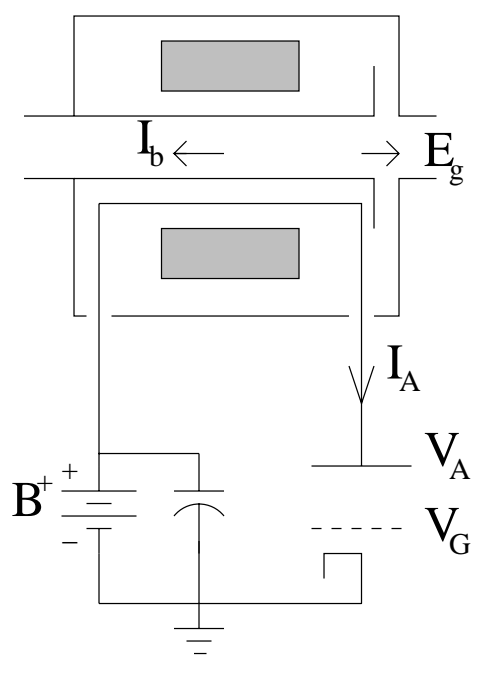

Figure 1: Equivalent beam-cavity circuit.

the feedback matrix assume that the error is small and define the error terms $\Delta I_{G}, \Delta I_{g}, \Delta Q_{G}, \Delta Q_{g}$. Inserting these errors in equation (1) and keeping first order terms yields

$$
\left(\begin{array}{c}
\Delta I_{G} \\
\Delta Q_{G}
\end{array}\right)=\frac{n_{g a p} R_{A}}{\mu\left|Z_{\ell}\right|}\left(\begin{array}{cc}
\cos \phi_{z} & -\sin \phi_{z} \\
\sin \phi_{z} & \cos \phi_{z}
\end{array}\right)\left(\begin{array}{c}
\Delta I_{g} \\
\Delta Q_{g}
\end{array}\right)
$$

where $R_{A}$ is the anode resistance and $Z_{\ell}$ is the loaded impedance of the cavity. The phase angle is given by

$$
\tan \phi_{z}=\frac{R_{\ell}}{\omega_{r f f} L_{g}}-\omega_{r f} C_{g} R_{\ell},
$$

where $R_{\ell}$ is the loaded shunt impedance of the gap. For feedback loops define a low level gain $G_{g}$. The low level loops cause the grid drive signal to change according to

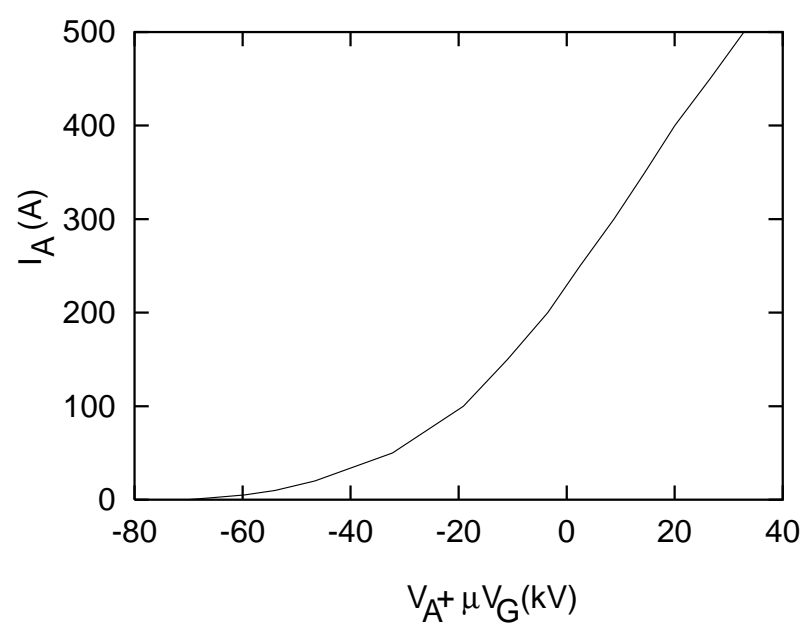

Figure 2: Anode current versus effective voltage for a screen voltage of $2 \mathrm{kV}$. The amplification factor is $\mu=132$

$$
\frac{d}{d n}\left(\begin{array}{c}
I_{G} \\
Q_{G}
\end{array}\right)=G_{g}\left(\begin{array}{cc}
\cos \phi_{z} & -\sin \phi_{z} \\
\sin \phi_{z} & \cos \phi_{z}
\end{array}\right)\left(\begin{array}{c}
\hat{I}_{g}-I_{g} \\
\hat{Q}_{g}-Q_{g}
\end{array}\right) .
$$

The phase angle is zero in the baseline design but, anticipating dynamic tuning, take it to be given by equation (7) with $R_{\ell}$ replaced by a constant. Assuming that $L_{g}(n)$ and and $C_{g}$ are reasonably well known, all the parameters of the voltage feedback loop are defined.

Along with the voltage feedback the design calls for a feed-forward correction using the measured beam current. Assume the in phase $I_{B}$ and quadrature $Q_{B}$ components of the beam current are measured using the same algorithm as for the in phase and quadrature components of the gap voltage. Let $r_{B}$ and $n_{B}$ be the accumulation rate and delay for the acquisition of the beam current components. The feed forward correction for the beam current is taken to be

$$
\left(\begin{array}{c}
I_{G}^{1} \\
Q_{G}^{1}
\end{array}\right)=G_{f} \frac{n_{g a p} R_{A}}{\mu}\left(\begin{array}{c}
I_{B}-\hat{I}_{B} \\
Q_{B}-\hat{Q}_{B}
\end{array}\right)
$$

where $\hat{I}_{B}$ and $\hat{Q}_{B}$ are the in phase and quadrature components of beam current for which the cavity is tuned. For an accurate value of $R_{A}$ the ideal feed forward gain is $G_{f}=1$. Let $I_{G}^{2}$ and $V_{G}^{2}$ be the solutions to equation (8) then, with the feed forward correction given by equation (9) the high frequency grid drive signal is given by

$$
\begin{aligned}
& V_{G}(t)=\left(I_{G}^{1}+I_{G}^{2}+I_{G}^{0}\right) \sin \left(\omega_{r f} t\right) \\
& \quad-\left(Q_{G}^{1}+Q_{G}^{2}+Q_{G}^{0}\right) \cos \left(\omega_{r f} t\right)+\bar{V}_{G},
\end{aligned}
$$

where $I_{G}^{0}$ and $Q_{G}^{0}$ are best guess values for the amplitude functions which are based on the behavior during earlier cycles. The in phase and quadrature amplitudes are defined at the beginning of each turn and linearly interpolated between turns.

The RF simulation uses standard techniques[9]. The previous equations are transformed into a set of first order differential equations and discretized. The total RF kick is updated once per particle per turn with 360 time slices per turn. Space charge makes a significant contribution to the longitudinal force. For frequencies below $f_{r f}^{2} / 4 \pi h f_{s} \sim 75 \mathrm{MHz}$ the space charge forces can be updated once per turn. At the reference azimuth the voltage due to space charge is

$$
V_{s c}(t)=\frac{Z_{s c}}{\omega_{0}} \frac{d I_{b}(t)}{d t},
$$

where $Z_{s c} \approx 200 \Omega$ is the space charge impedance[10]. The space charge kick was obtained by convolving the binned beam current with a differentiated, $50 \mathrm{~ns}$ pulse. The beam is modeled by $M \sim 10^{4}$ macro-particles. The beam current on $n$th turn is a sum over macro-particles and a binning/smoothing function $f(t)$ is used to reduce unphysical high harmonics

$$
I_{b}(t)=\sum_{k=1}^{M} f\left(t-t_{n}^{k}\right) .
$$


The change in proton energy depends on arrival time at the gap.

$$
E_{n+1}^{k}=E_{n}^{k}+q V_{\text {total }}\left(t_{n}^{k}\right),
$$

where the total voltage is $n_{\text {cau }} n_{\text {gap }} V_{\text {gap }}$ plus 2 nd harmonic and space charge. Arrival time on the next turn changes depending on the energy deviation

$$
t_{n+1}^{k}=t_{n}^{k}+\eta T_{\text {rev }} \frac{E_{n+1}^{k}-E_{0}}{E_{0} \beta^{2}} .
$$

The simulations used the parameters in Table 1. Notice that the injected energy spread is $\pm 3.8 \mathrm{MeV}$ with a rectangular distribution. This is created by an energy wiggler cavity running at a slightly different frequency from the LINAC RF. The net effect is to broaden the rms width of the energy distribution without creating the tails associated with a debuncher cavity. The simulations assumed 2 turns of delay and the accumulation rates $r_{g}$ and $r_{B}$ were taken to be $100 / \mathrm{ms}$. The low level gain $G_{g}$ was chosen to give a comparable transient. The target $h=1 \mathrm{RF}$ voltage was ramped from 30 to $40 \mathrm{kV}$ over the first 500 turns. To be pessimistic, the best guess values of the in phase and quadrature grid drive voltages were nulled $I_{G}^{0}=Q_{G}^{0} \equiv 0$. Only the voltage error and feed forward signals, via equations (8) and (9), created the grid drive voltage for $h=1$. With its smaller beam loading, the $h=2$ voltage was set to half the $h=1$ target value throughout the cycle.

Figure 3 shows the longitudinal phase space, the rf bucket, and the edges of the kicker gap just before extraction. Figure 4 shows the bunch current, the space charge voltage and the total RF voltage just before extraction. The gap is clean and the bunching factor is 0.4 .

Figure 5 illustrates the situation when there is no feed forward. The maximum error in the RF voltage waveform is $\lesssim 5 \mathrm{kV}$ and the bunch is acceptable. However, with the heavy beam loading, feedforward is required for system stability and a small, unstable, oscillation exists without it. Additionally, a feed forward system will reduce the deleterious impact of $e . g$. variations due to aging of the tetrode. In practice, even with marginal feed forward the RF system should easily meet its specifications.

\section{DYNAMIC TUNING}

By changing the cavity resonant frequency as the beam is stacked the amount of RF drive current can be reduced. Conversely, the $h=1$ gap voltage can be increased. For a steady state system with beam current $I_{b}(t)=\hat{I}_{b} \cos \left(\omega_{r f} t\right)$ and gap voltage $V_{g}(t)=\hat{V}_{g} \sin \left(\omega_{r f} t\right)$, the minumum RF anode current is $I_{A}(t)=\left(n_{g a p} \hat{V}_{g} / R_{f}\right) \sin \left(\omega_{r f} t\right)$ and the cavity resonant frequency is

$$
\omega_{\text {res }}=\omega_{r f f}\left(1+\frac{\hat{I}_{b}}{\hat{V}_{g} C_{g} \omega_{r f}}\right)^{1 / 2} .
$$

Since $\omega_{\text {res }}=1 / \sqrt{L_{g} C_{g}}$ the ideal frequency can be obtained by changing the cavity inductance. This is accomplished by changing the bias field within the ferrites and is

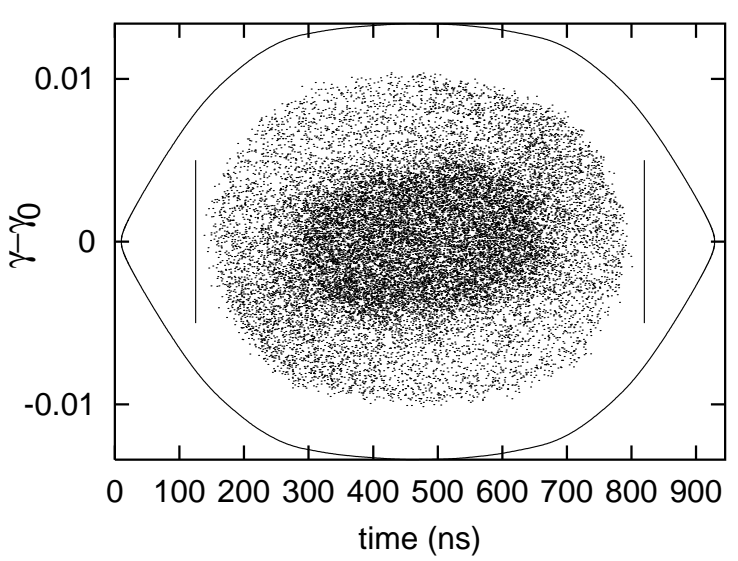

Figure 3: Phase space distribution and bucket at extraction for nominal design.

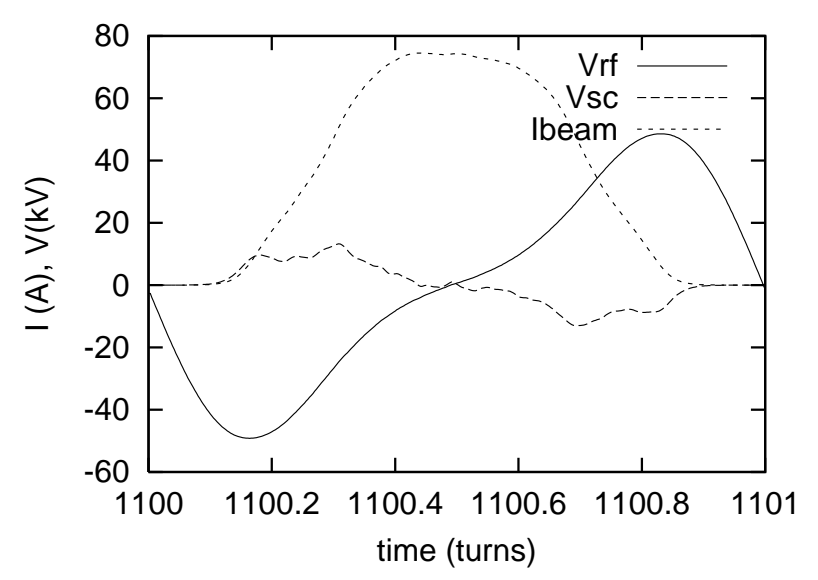

Figure 4: Currents and voltages at extraction for nominal design. The binned current, convolved with a 50ns smoothing pulse, is shown.

refered to as tuning the cavity. Dynamic tuning refers to continuously changing the ferrite bias so that equation (12) is satisfied throughout the cycle. For SNS with $2 \times 10^{14}$ protons and $\hat{V}_{g}=6.7 \mathrm{kV}$, the inductance per gap needs to drop from $7.5 \mu \mathrm{H}$ to $5.7 \mu \mathrm{H}$ during $1 \mathrm{~ms}$. The RF magnetic field will be $\sim 300 \mathrm{G}$ so the ferrites are changing quickly under high field conditions. Experiments are needed to test this scenario.

Experiments have been done on a parallel LRC circuit. The inductance was provided by two ferrite cores with 4 RF coupling turns, and the cores were in parallel. A $1 \mathrm{k} \Omega$ resistor, and a $2.9 \mathrm{nF}$ of capacitor completed the circuit. For no tuning current the measured inductance of the ferrites measured in parallel $L=9.8 \mu \mathrm{H}$. This gives a bare resonant frequency of $944 \mathrm{kHz}$. The drive frequency was swept from $1.092 \mathrm{MHz}$ to $1.249 \mathrm{MHz}$ over $1 \mathrm{~ms}$ and the system was pulsed at $60 \mathrm{~Hz}$.

With a constant bias current, and a nearly constant drive current amplitude, the RF voltage dropped from $390 \mathrm{~V}$ to $65 \mathrm{~V}$ during the frequency sweep. When the bias current 


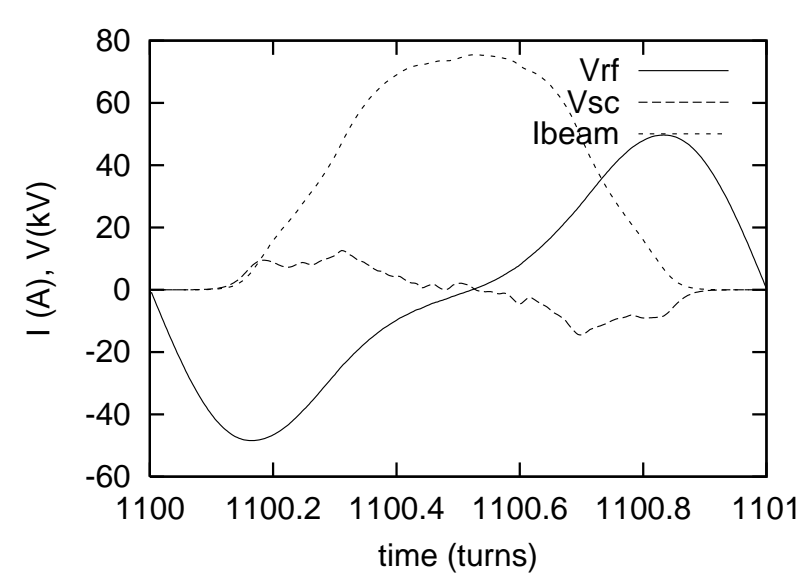

Figure 5: Currents and voltages at extraction for no feed forward.The binned current, convolved with a $50 \mathrm{~ns}$ smoothing pulse, is shown.

was ramped linearly during the sweep, with the same drive current amplitude, the RF voltage remained nearly constant at $275 \mathrm{~V}$. Optimal results required the bias current to be reversed between RF pulses to reset the ferrite hysteresis curve. The reduction in peak voltage is due to dynamic loss within the ferrite and can be compensated by increasing the drive.

With four coupling turns and $300 \mathrm{~V}$, these experiments test an equivalent gap voltage of $1.6 \mathrm{kV}$. Further tests are needed to check operating conditions. Under the assumption that the ferrite tests will succeed, simulations have included dynamic tuning. Figures 6 and 7 show its clear benefits.

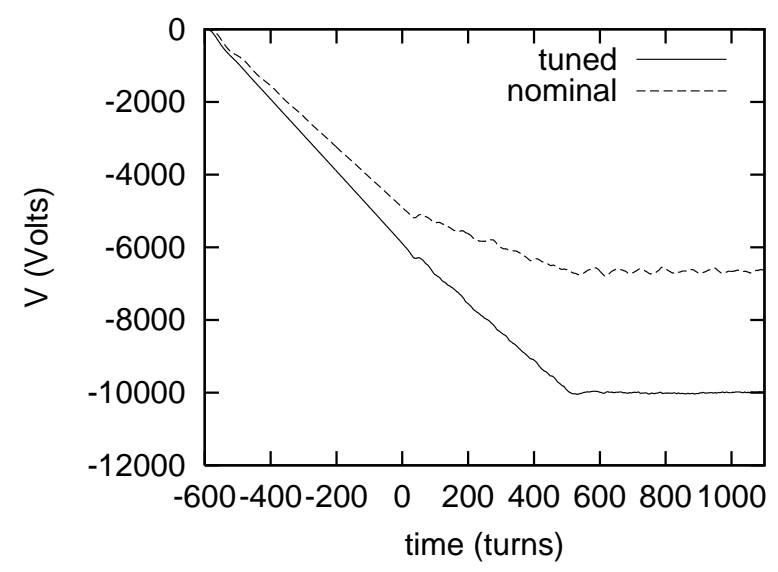

Figure 6: In phase amplitude of gap voltage $\left(I_{g}\right)$ during the cycle for the nominal case and with dynamic tuning.

In both cases, the RF drive starts turning on 600 turns before injection starts (turn 0 ). For the nominal case the inductance is held constant at $7.5 \mu \mathrm{H}$ while for the tuned case the inductance varies linearly with time. At turn 0 the tuned inductance is $L_{g}=7.5 \mu \mathrm{H}$ while at turn 1100 (extraction) it is $L_{g}=6.2 \mu \mathrm{H}$. The $h=1$ gap voltage in the tuned case corresponds to $60 \mathrm{kV} /$ turn as compared

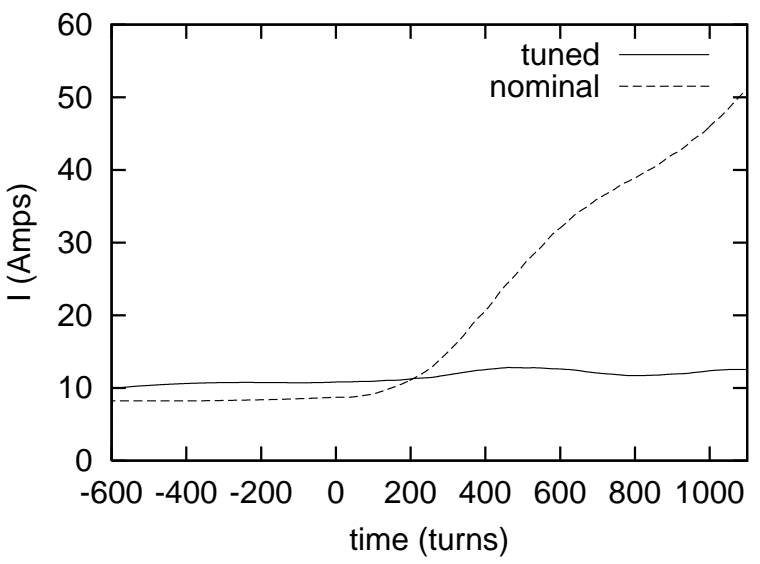

Figure 7: DC anode current during the cycle for the nominal case and with dynamic tuning.

with the nominal $40 \mathrm{kV} /$ turn. The $h=2$ voltage ramped from $15 \mathrm{kV}$ to $20 \mathrm{kV}$ in both cases. The average anode current is significantly smaller for the tuned case. This is true even though the anode power supply voltage is $B^{+}=$ $12 \mathrm{kV}$ for the tuned case and $B^{+}=10 \mathrm{kV}$ for the nominal case. Additionally, the total energy deposited in the anode each SNS cycle is significantly smaller for the tuned case.

\section{ACKNOWLEDGEMENTS}

Many people have contributed to this work. In particular we thank D. Boussard, Y.Y. Lee, D. Raparia, K. Rogers, R. Spitz, and R. Sanders.

\section{REFERENCES}

[1] National Spallation Neutron Source, Conceptual Design Report, 4-64, (1997).

[2] J. Wei, 7th European Particle Accelerator Conference,pg 123, (2000).

[3] M. Blaskiewicz, J.M. Brennan, Y.Y. Lee SNS tech note \# 9, (1996).

[4] M. Blaskiewicz, J.M. Brennan, A. Zaltsman SNS tech note \# 36, (1997).

[5] M. Blaskiewicz, J.M. Brennan, J. Brodowski, J. Delong, M. Meth, E. Onillon, A. Zaltsman , 7th European Particle Accelerator Conference,pg 1942, (2000).

[6] F. Pederson, IEEE, TNS, Vol. NS-22, No. 3, pg 1906, (1975).

[7] R. Garoby, Fontiers of Particle Beams: Intensity Limitations, US-CERN School on Particle Accelerators, Springer-Verlag, pg 509, (1990).

[8] D. Boussard, CERN 91-04 (1991).

[9] S. Koscielniak, TRI-DN-97 (1997) and references therein

[10] S.Y. Zhang, SNS Tech Note \# 43 (1999). 